\title{
A Decoupled Non-SSA Global Register Allocation Using Bipartite Liveness Graphs
}

\author{
RAJKISHORE BARIK, Intel Labs \\ JISHENG ZHAO and VIVEK SARKAR, Rice University
}

Register allocation is an essential optimization for all compilers. A number of sophisticated register allocation algorithms have been developed over the years. The two fundamental classes of register allocation algorithms used in modern compilers are based on Graph Coloring (GC) and Linear Scan (LS). However, these two algorithms have fundamental limitations in terms of precision. For example, the key data structure used in GC-based algorithms, the interference graph, lacks information on the program points at which two variables may interfere. The LS-based algorithms make local decisions regarding spilling, and thereby trade off global optimization for reduced compile-time and space overheads. Recently, researchers have proposed Static Single Assignment (SSA)-based decoupled register allocation algorithms that exploit the live-range split points of the SSA representation to optimally solve the spilling problem. However, SSA-based register allocation often requires extra complexity in repairing register assignments during SSA elimination and in addressing architectural constraints such as aliasing and ABI encoding; this extra overhead can be prohibitively expensive in dynamic compilation contexts.

This article proposes a decoupled non-SSA-based global register allocation algorithm for dynamic compilation. It addresses the limitations in current algorithms by introducing a Bipartite Liveness Graph (BLG)-based register allocation algorithm that models the spilling phase as an optimization problem on the BLG itself and the assignment phase as a separate optimization problem. Advanced register allocation optimizations such as move coalescing, live-range splitting, and register class handling are also performed along with the spilling and assignment phases. In the presence of register classes, we propose a bucket-based greedy heuristic for assignment that strikes a balance between spill-cost and register class constraints. We present experimental evaluation of our BLG-based register allocation algorithm and compare it with production-quality register allocators in Jikes RVM and LLVM.

Categories and Subject Descriptors: D.3.4 [Programming Languages]: Processors

General Terms: Algorithms, Performance

Additional Key Words and Phrases: Decoupled register allocation, bipartite graph, coalesce graph, basic and compound intervals

\section{ACM Reference Format:}

Barik, R., Zhao, J., and Sarkar, V. 2013. A decoupled non-SSA global register allocation using bipartite liveness graphs. ACM Trans. Architec. Code Optim. 10, 4, Article 63 (December 2013), 24 pages.

DOI: http://dx.doi.org/10.1145/2544101

\section{INTRODUCTION}

Register allocation is an essential compiler optimization that has received much attention from the research community during the past five decades. Its relevance continues to increase with current trends toward energy-efficient processors in which some of the

Authors' addresses: R. Barik, Intel Labs, 2200 Mission College Blvd. Santa Clara, CA 95054, USA; email: rajkishore.barik@intel.com; J. Zhao and V. Sarkar, Computer Science Department, Rice University, Houston, TX 77005, USA; emails: Jisheng.Zhao@rice.edu, vsarkar@rice.edu.

Permission to make digital or hard copies of part or all of this work for personal or classroom use is granted without fee provided that copies are not made or distributed for profit or commercial advantage and that copies show this notice on the first page or initial screen of a display along with the full citation. Copyrights for components of this work owned by others than ACM must be honored. Abstracting with credit is permitted. To copy otherwise, to republish, to post on servers, to redistribute to lists, or to use any component of this work in other works requires prior specific permission and/or a fee. Permissions may be requested from Publications Dept., ACM, Inc., 2 Penn Plaza, Suite 701, New York, NY 10121-0701 USA, fax +1 (212) 869-0481 or permissions@acm.org.

(c) 2013 ACM 1544-3566/2013/12-ART63 $\$ 15.00$

DOI: http://dx.doi.org/10.1145/2544101 
burden of memory hierarchy management is shifting back from hardware to software. Two fundamental classes of register allocation algorithms have emerged over the years: Graph Coloring (GC) and Linear Scan (LS). Register allocation algorithms based on GC [Chaitin et al. 1981; Briggs et al. 1994; George and Appel 1996; Park and Moon 1998; Smith et al. 2004], including more recent variants based on Static Single Assignment (SSA) form [Hack and Goos 2006], all use the Interference Graph (IG) as a primary data structure. Although the IG captures interferences among live ranges precisely, its lack of program point specific information can lead to imprecise results, especially for scenarios where insertion of additional move and exchange instructions can avoid spilling. On the other hand, register allocation algorithms based on LS [Traub et al. 1998; Poletto and Sarkar 1999; Wimmer and Mössenböck 2005; Sarkar and Barik 2007; Wimmer and Franz 2010] overcome the compile-time and compile-space overheads of GC algorithms, but do so at the expense of achieving poorer execution times than GC. The key reason for this is due to the lack of global information while making the spilling decisions. The primary goal of this article is to address these limitations using a program point specific data structure called the Bipartite Liveness Graph (BLG).

A secondary goal of this article is to simplify the implementation of the register allocator by decoupling the register spilling and register assignment phases in an optimizing back end. This will allow the spilling phase to focus on spilling decisions and the assignment phase to focus on coalescing and physical register assignment decisions. Although this form of decoupling has been performed for other register allocation algorithms in the past, including the work of Appel and George [2001] and SSA-based register allocation algorithms [Hack and Goos 2006; Brisk 2006; Pereira and Palsberg 2009; Colombet et al. 2011], our approach is unique in its use of the BLG for the spilling phase and the Coalesce Graph (CG) for the assignment phase. The CG consists of both IR move instructions and register-to-register moves that arise from our BLG-based allocation phase. In GC algorithms, the coupling between these phases is manifest in the integration of coloring and coalescing decisions, which can further compromise the effectiveness of the final solution and complicate the implementation of the allocator. These complications arise from nontrivial problems that must be addressed by the implementer in dealing with coalescing in traditional GC allocators. Further, register allocation for today's architectures includes new challenges due to hardware features such as register classes, register aliases, precoloring, and register pairs. To produce high-quality machine code, a register allocator must consider these hardware features in both the allocation and assignment phases.

Although recent trends in register allocation are shifting toward decoupled SSAbased algorithms, there are known complexities in SSA elimination after register allocation [Brisk 2006; Pereira and Palsberg 2009] and in addressing architecture-level register aliasing and encoding constraints [Colombet et al. 2011] suggesting that the decoupled SSA approach will be challenging to use for dynamic compilation.

This article addresses the register allocation challenges listed earlier by starting with a clean separation between the register spilling and register assignment phases. The spilling phase is modeled as an optimization problem on a new data structure: the BLG. As we will see, the BLG is a more precise data structure than the IG. Assignment is modeled as a separate optimization problem that incorporates register-to-register moves and exchanges as alternatives to spilling and handles move coalescing and register class constraints.

Specifically, we make the following contributions toward the mentioned goals:

(1) We introduce a novel BLG representation as an alternative to the IG representation.

(2) We formulate the spilling problem for BLGs as a simple optimization problem and present a greedy heuristic to solve it. The spilling phase is performed independently 
of coalescing optimizations. We also extend the spilling phase to support partial spills.

(3) We formulate spill-free register assignment with move coalescing as a combined optimization problem that maximizes the benefits of move coalescing while finding an assignment for every symbolic register. Move coalescing is performed on a CG. A local greedy heuristic is presented to address the assignment optimization problem.

(4) We extend the register assignment approach from (3) to handle register classes. An optimized version of the assignment problem is presented that minimizes the additional spilled symbolic registers and, at the same time, maximizes the benefits of move coalescing. A prioritized bucket-based greedy heuristic is presented to address this problem.

\section{BIPARTITE LIVENESS GRAPH}

We start this section with some definitions. A program point can be split into two program points based on the values read and written at that program point [Sarkar and Barik 2007]:

Definition 2.1. Each program point $p$ is split into $p^{-}$and $p^{+}$, where $p^{-}$consists of the variables that are read at $p$ and $p^{+}$consists of the variables that are written at $p$.

$[x, y]$ is called a basic interval for variable $v$ (denoted as $\mathrm{BI}(v)$, for a basic interval of $v$ ) if and only if for every program point, $p$, such that $p \geq x$ and $p \leq y$ imply $v$ is live at $p$. Note that $\mathrm{BI}(v)$ does not include any hole. $x$ and $y$ denote the start and end points of $\mathrm{BI}(v)$, respectively. A compound interval for a variable $v$ (denoted as $\mathrm{CI}(v)$ ) consists of a set of basic intervals for $v . \mathrm{CI}(v)$ can have holes. Let $\mathcal{B}$ denote the set of all basic intervals and $\mathcal{C}$ denote the set of all compound intervals in the program. Let $\mathcal{L}$ denote the set of start points and $\mathcal{H}$ denote the set of end points of all the basic intervals.

The number of simultaneously live symbolic registers at a program point $p$ is denoted by numlive $(p)$. MAXLIVE represents the maximum number of simultaneously live symbolic registers in any program point. A program point $p$ is said to be constrained if numlive $(p)>k$, where $k$ is the total number of machine registers. In the presence of register classes, we call a program point $p$ constrained if it violates any of the register requirements of any of the register classes of the symbolic registers that are live at $p$.

Now we present a new representation, BLG, which captures program point-specific liveness information as an alternative to the IG.

Definition 2.2. Bipartite Liveness Graph: A BLG is a undirected weighted bipartite $^{1}$ graph $G=\langle U \cup V, E\rangle$, where $V$ denotes all of the basic interval end points ${ }^{2}$ in $\mathcal{H}$, $U$ denotes all the compound intervals in $\mathcal{C}$, and an edge $e=(u, v) \in E$ indicates that the compound interval $u \in U$ is live at the interval end point $v \in V$. Each $u \in U$ has an associated nonnegative weight $S P I L L(u)$ that denotes the spill cost of $u$. Similarly, each $v \in V$ has an associated nonnegative weight $F R E Q(v)$ that denotes the execution frequency of the $I R$ instruction associated with basic interval end point $v$.

Obviously, it is a waste of space to capture liveness information at every program point in $V$ of BLG. From a register allocation perspective, it suffices to consider only constrained program points corresponding to either the basic interval start points alone or end points alone but not both in $V$. This is because spilling/assignment decisions only need to be taken at those points. Additional optimizations are possible as well-for example, if two interval end points have the same liveness information (i.e., same set

\footnotetext{
${ }^{1} \mathrm{~A}$ bipartite graph is a graph whose vertices can be divided into two disjoint sets $U$ and $V$ such that each edge connects a vertex in $U$ to one in $V$.

${ }^{2}$ The choice of interval end points is arbitrary. We could have used interval start points instead.
} 
(a) Code fragment with basic and compound intervals:

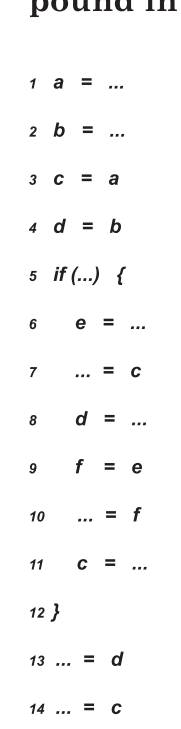

(b) Interference Graph (dashed

lines show move instructions):

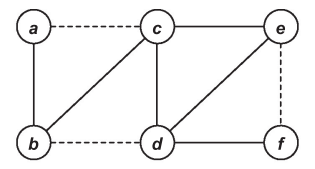

(c) Bipartite Liveness Graph (BLG) (with unconstrained end points):

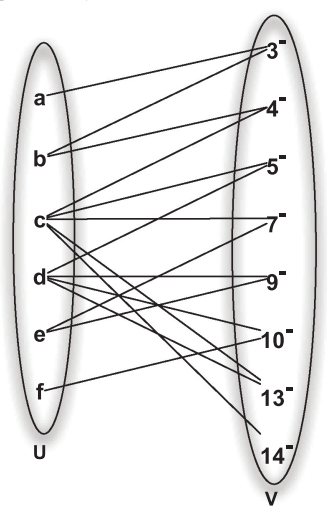

Fig. 1. (a) Example code fragment with basic and compound intervals; the dotted lines represent end points of basic intervals. (b) IG; the solid lines represent interference, and the dashed lines represent move instructions. (c) BLG with unconstrained interval end points; the vertices on the left of the graph represent compound intervals, and the vertices on the right represent basic interval end points. With two physical registers, the BLG representing constrained end points is empty in this case.

of variables live), only one of them (but not both) needs to be added to the BLG for spilling decisions.

Figure 1 presents an example code fragment with its basic and compound intervals in Figure 1(a) and the IG in Figure 1(b). We observe that IG has a clique of size 3 due to the cycle comprising nodes $c, d$, and $e$. Now consider a GC register allocator that performs coalescing along with register allocation. Both aggressive [Chaitin et al. 1981] and conservative [Briggs et al. 1994] coalescing will be able to eliminate the move edges $(a, c),(b, d)$, and $(e, f)$ without increasing the colorability of the original IG. If we have two physical registers, we have to spill one of the coalesced nodes $a c, b d$, and $e f$. The uncoalescing approach used in an optimistic coalescing technique [Park and Moon 1998] will be able to just spill one of the nodes involved in the cycle as it tries all possible combinations of assigning colors to individual nodes of a potentially spilled coalesced node. The points to note here are that we cannot color the IG using two physical registers and that opportunities for coalescing can be missed due to the inability to color certain nodes.

A closer look at the code reveals the fact that none of the program points has more than two variables live simultaneously. If this is the case, two questions come to mind: (1) Can we generate spill-free code with two physical registers that does not give up any coalescing of symbolic registers? (2) If the answer to the first question is yes, then why did GC generate spill code and also miss the coalescing opportunity?

The answer to the first question is yes. The BLG with unconstrained interval end points for the example code is shown in Figure 1(c). This captures the fact that every basic interval end point in $V$ has degree less than or equal to 2, 


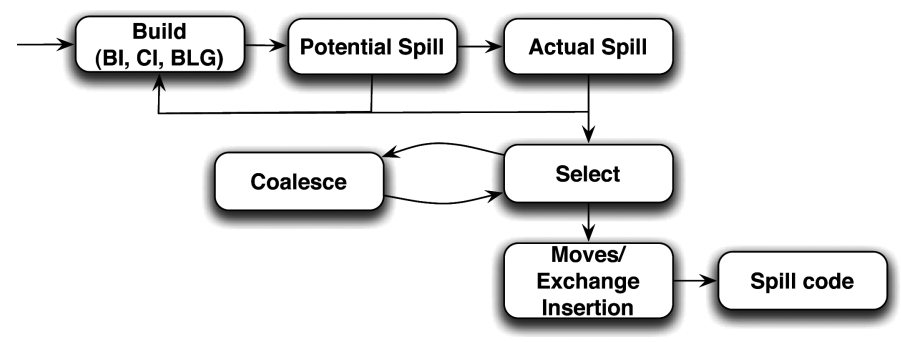

Fig. 2. Register allocation using Bipartite Liveness Graph.

indicating that no more than two compound intervals are simultaneously live. (The BLG with constrained interval end points is empty in this case.) Let us name the two physical registers as $r_{1}$ and $r_{2}$. The following register assignment is possible: $\operatorname{reg}\left(\left[1^{+}, 3^{-}\right]\right)=r_{1}, \operatorname{reg}\left(\left[2^{+}, 4^{-}\right]\right)=r_{2}, \operatorname{reg}\left(\left[4^{+}, 5^{-}\right]\right)=r_{2}, \operatorname{reg}\left(\left[3^{+}, 7^{-}\right]\right)=r_{1}$, $\operatorname{reg}\left(\left[6^{+}, 9^{-}\right]\right)=r_{2}, \operatorname{reg}\left(\left[9^{+}, 10^{-}\right]\right)=r_{2}, \operatorname{reg}\left(\left[8^{+}, 13^{-}\right]\right)=r_{1}$, and $\operatorname{reg}\left(\left[11^{+}, 14^{-}\right]\right)=r_{2}$. This register assignment requires an additional register exchange operation since the register assignment for the basic intervals of both $\mathrm{CI}(c)$ and $\mathrm{CI}(d)$ were exchanged when the code after the if condition was executed. We need to insert an exchg $r_{1}, r_{2}$ instruction on the control flow edge between 4 and 13. As a result, no coalescing opportunities in lines 3,4 , and 9 were given up during such an assignment.

Now let us try to answer the second question. Looking at the code fragment, we observe that at the program point $13^{-}, d$ interferes with two values of $c$ that are assigned on lines 3 and 11. Similarly, $c$ interferes with two values of $d$ that are assigned on lines 4 and 8. During runtime, if the if branch is taken, then assignments on lines 8 and 11 will be visible to the code following the if condition; otherwise, assignments on lines 3 and 4 will be visible. This notion can not be precisely captured using the definition of live ranges in an IG unless we convert the program to SSA form or perform liverange splitting [Appel and George 2001]. Each of these approaches requires additional complexities (e.g., the SSA-based approach needs to handle out-of-SSA translation by inserting extra copy statements).

The previous example raises a question about the general approach of stating the global register allocation problem as the GC problem on the IG. Although the IG using live ranges provides a global view of the program, it is less precise than a BLG with intervals.

Similar to GC, an LS register allocation algorithm (e.g., the LS algorithm implemented in Jikes RVM) when applied to Figure 1 will first spill one of $\operatorname{CI}(c), \operatorname{CI}(d)$, or $\mathrm{CI}(e)$ compound intervals decided based on spill cost. If it decides to spill $\mathrm{CI}(e)$, then later on it will force another spill to one of $\mathrm{CI}(c), \mathrm{CI}(d)$, or $\mathrm{CI}(f)$. This scenario is even worse than GC as it may spill more than one compound intervals. This problem arises in LS primarily due to the local decisions taken during the combined spilling and assignment phase.

\section{OVERALL APPROACH}

The overall register allocator presented in this article is depicted in Figure 2. The first step in the allocator is to build data structures for basic intervals, compound intervals, and the BLG. Then, the spilling is performed on the BLG to determine a set of compound intervals that need to be spilled as shown in the blocks for potential spill and actual spill. A combined phase of assignment and coalescing is then performed until all of the symbolic registers are assigned physical registers or spilled. Next, register move and 
exchange instructions are added to the $I R$ to produce correct code. Finally, spill code is added to the $I R$.

\section{SPILLING USING BIPARTITE LIVENESS GRAPHS}

In this section, we first describe an all-or-nothing approach for spills-that is, if a symbolic register is selected for spilling, every access of the symbolic register in the program will be replaced by a load or store instruction. Extending the BLG to partial spills is described later in Section 4.1.

Definition 4.1. Spill Optimization Problem: Given a BLG with constrained end points, $G$, and $k$ uniform physical registers, find a spill set $S \subseteq U$ and $G^{\prime} \subseteq G$ induced by $S$ such that (1) $\forall v \in V, v$ is unconstrained-that is, $D E G R E E(v) \leq k$; and (2) $\sum_{s \in S} S P I L L(s)$ is minimized. For each compound interval $s \in S$ and basic interval $b \in s$, set spilled $(b):=$ true.

Given a BLG, the spill decision problem now reduces to an optimization problem whose solution ensures that no more than $k$ physical registers are needed at every interval end point, and at the same time, spills as few compound intervals as possible. Algorithm 1 provides a greedy heuristic that solves the spill optimization problem. Steps 3 through 11 choose Potential Spill candidates (as shown in Figure 2) using a maxmin heuristic. Each iteration of the loop alternates between largest frequency interval end point and smallest spill cost symbolic register. The alternating approach allows the option of completely unconstraining a high-pressure region of program points before moving onto another. Steps 12 through 15 unspill some of the potential spill candidates, resulting in Actual Spill (as shown in Figure 2) candidates. The unspilling step reverts a potential spill candidate and its edges back onto the BLG and verifies if the BLG becomes constrained after adding the potential spill candidate. If the BLG does not get constrained, then the symbolic register can be unspilled. Depending on the quality of potential spill candidate selection, the unspilling of spill candidates provides a way of rectifying the obvious spilling mistakes (akin to unspilling in GC). The examination order of unspilling can have impact on final spilling decisions-currently, we use a stack data structure that orders the potential spill symbolic registers in nonincreasing spill cost.

One of the advantages of Algorithm 1 is that if a spill-free allocation exists, the algorithm is guaranteed to find an allocation without spills. On the other hand, if one works with an allocator based on GC, it is an NP-hard problem to determine if a spill-free allocation exists. This seeming contradiction arises because BLG may require the insertion of register-copy instructions (described in Section 5), whereas the standard GC algorithm does not allow for this possibility. Prior work on SSA-based register allocation [Hack and Goos 2006; Brisk et al. 2005; Bouchez 2009] and on Extended Linear Scan (ELS) [Sarkar and Barik 2007] independently established that the existence of a spill-free allocation can be determined in polynomial time, provided that extra register-copy instructions can be inserted. In the case of SSA-based register allocation, the extra copies arise from $\phi$-functions; in the case of ELS, they arise from the need to map from the register assignment for a symbolic register to another on a control flow edge. In both cases, the task of optimizing the additional copy instructions is a nontrivial problem.

Theorem 4.2. Algorithm 1 ensures that every program point has $k$ or fewer symbolic registers simultaneously live.

Proof: First, we need to prove that Algorithm 1 makes every node $v \in V$ unconstrained. This is trivial, as the algorithm continues to execute the while loop in Steps 4 


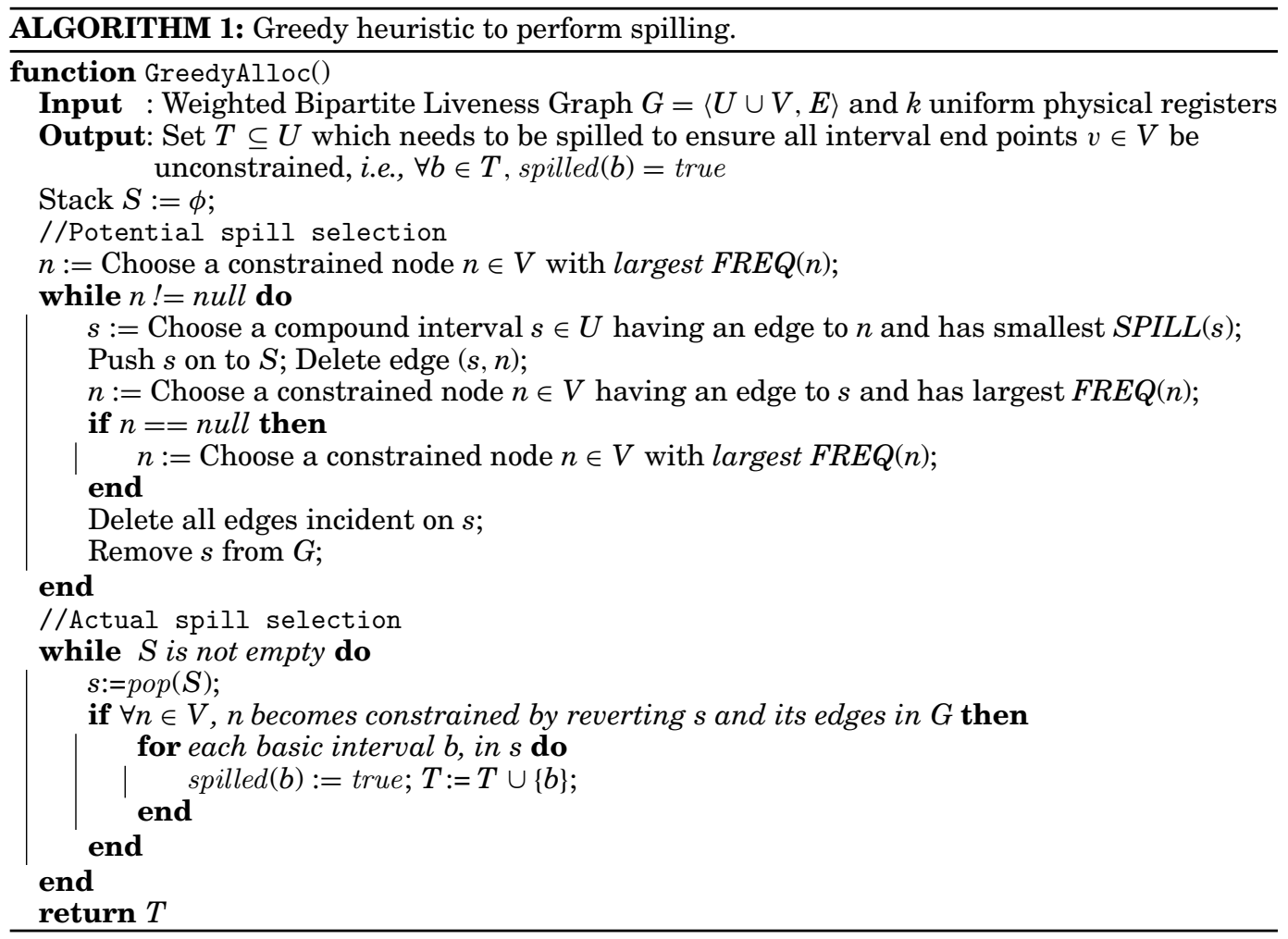

through 11 until there are constrained nodes $v \in V$ in the BLG. This is guaranteed by Steps 3, 7, and 9 in Algorithm 1. Second, we need to show that if no $v \in V$ is constrained, then every program point is unconstrained. We can prove this by contradiction. That is, if a program point, say $p^{+}$, is still constrained after all $v \in V$ are unconstrained, then we prove that such $p^{+}$does not exist. Obviously, $p^{+}$cannot be an end point. Let $n^{-}$represent the immediate next interval end point in the linear order of instructions from $p^{+}$. Thus, all program points from $\left[p^{+}, n^{-}\right]$are constrained. This implies that $n^{-}$ must be constrained. This is a contradiction, since $n^{-}$is an interval end point and is constrained. Hence proved.

THEOREM 4.3. Given the bipartite liveness graph, Algorithm 1 requires $\mathcal{O}(|\mathcal{H}| *$ $\max (0,(M A X L I V E-k)) *|\mathcal{C}|)$ time.

Proof: Every interval end point in $\mathcal{H}$ is traversed at most MAXLIVE $-k$ number of times to make it unconstrained. To make an interval end point unconstrained, we need to visit all of its neighbor and choose a minimum spill cost compound interval. This requires, at most, $|\mathcal{C}|$ edge visits.

\subsection{Bipartite Liveness Graphs with Partial Spills}

We now extend the register allocation problem for the BLG to allow for partial spillsthat is, for splitting a symbolic register so that it can be assigned to registers at some program points and accessed from memory at other program points. Live-range splitting has also been considered quite extensively in past work, although often with inconclusive results on the benefits of splitting. We consider a special case of partial spills, namely that of identifying one basic interval of a symbolic register for spilling. 
More general splitting of live ranges (as in Bergner et al. [1997] say) is a subject for future work.

For partial spills, we define SPILLBI for a basic interval that captures the spill cost of a basic interval including the cost for additional loads and stores for partial spilling. The problem statement can be summarized as follows.

Definition 4.4. Bipartite Liveness Graph with Partial Spills: A bipartite liveness graph with partial spills (BLGP) is a undirected weighted bipartite graph $G=\langle U \cup V, E\rangle$, where $V$ denotes all basic interval end points in $\mathcal{H}, U$ denotes all basic intervals in $\mathcal{B}$, and an edge $e=(u, v) \in E$ indicates that the basic interval $u \in U$ is live at the interval end point $v \in V$. Each $u \in U$ has an associated nonnegative weight $\operatorname{SPILLBI}(u)$ that denotes the spill cost of $u$. Similarly, each $v \in V$ has an associated nonnegative weight $F R E Q(v)$ that denotes the execution frequency of the $I R$ instruction associated with basic interval end point $v$.

Definition 4.5. Register Allocation Optimization Problem with Partial Spills: Given a BLG with constrained end points, $G$, and $k$ uniform physical registers, find a spill basic interval set $S \subseteq U$ and $G^{\prime} \subseteq G$ induced by $S$ such that (1) $\forall v \in V, v$ is unconstrained - that is, $D \bar{E} G R E E(v) \leq \bar{k}$; and (2) $\sum_{s \in S} S P I L L B I(s)$ is minimized. For each basic interval $s \in S$, set spilled $(b):=$ true.

Algorithm 1 can be extended easily to support partial spills. That is, Steps 3 through 11 can be modified to choose potential spill basic intervals instead of compound intervals using the original max-min heuristic. Similarly, the unspilling in Steps 12 through 15 rectifies the spilling decisions by resurrecting basic intervals instead of compound intervals.

\section{ASSIGNMENT USING REGISTER MOVES AND EXCHANGES}

The spilling phase ensures that every program point needs $k$ or fewer physical registers. In this section, we first describe how assignment for basic intervals can be performed by possibly adding extra register moves/exchanges to the $I R$ without spilling any symbolic registers.

\subsection{Spill-Free Assignment}

Definition 5.1. Spill-free Assignment: Given a set of basic intervals $b \in \mathcal{B}$ with spilled $(b)=$ false, and $k$ uniform physical registers, find register assignment $\operatorname{reg}(b)$ for every basic interval, $b \in \mathcal{B}$, including any register-to-register copy or exchange instructions that need to be inserted in the $I R$.

The algorithm to perform register assignment for basic intervals is provided in Algorithm 2. The algorithm sorts the basic intervals in increasing start points. Steps 4 through 11 perform assignment to basic intervals using an avail list of physical registers. The assignment to a basic interval first prefers getting the physical register that was previously assigned to another basic interval of the same compound interval (as shown in Step 7). This avoids the need for additional move/exchange instructions. However, in cases where the already assigned physical register is unavailable, we assign a new available physical register (as shown in Step 10). Assigning such a new physical register may produce incorrect code without additional move/exchange instructions on certain control flow paths.

Steps 12 through 20 of Algorithm 2 create a list of move instructions that need to be inserted on a control flow edge. These move instructions form the nodes of a directed anti-dependence graph $D$ in Algorithm 3. The edges in $D$ represent the anti-dependence between a pair of move instructions. Steps 5 through 10 of Algorithm 3 add the 


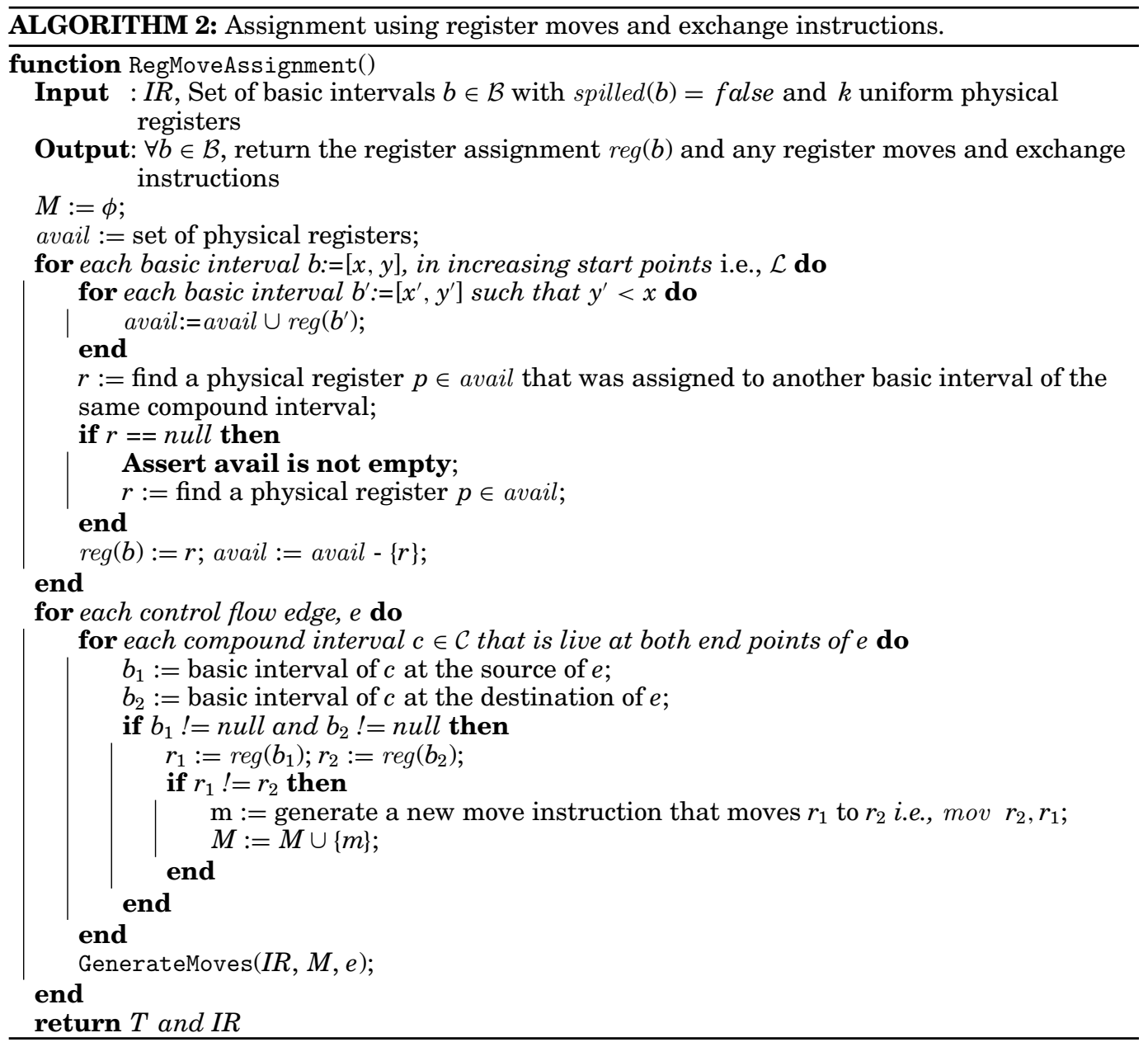

anti-dependence edges to $D$. A Strongly Connected Component (SCC) search is performed on $D$ to generate efficient code using exchange instructions for SCCs of size 2 or more (as shown in Steps 11 through 18). The nodes in an SCC are collapsed to a single node with exchange instructions. Finally, a topological sort order of $D$ produces the correct code for a control flow edge $e$.

\section{Lemma 5.2. The assertion on line 9 of Algorithm 2 never fails.}

Proof: Follows from the fact that every interval end point has no more than $k$ symbolic registers simultaneously live.

TheOREM 5.3. Spill-free assignment takes $\mathcal{O}\left(|\mathcal{E}| *\left(|\mathcal{C}|+|\mathcal{K}|^{2}\right)\right)$ space, where $\mathcal{E}$ represents the control flow edges in a program and $\mathcal{K}$ represents the available physical registers.

Proof: Additional space requirement in assignment phase is due to the antidependence graph $D$. For every control flow edge $e \in \mathcal{E}$, in the worst case we need to insert $|\mathcal{C}|$ number of register-to-register move instructions. These are the number of nodes in $D$. The number of edges in $D$ are bounded by the square of physical registers 


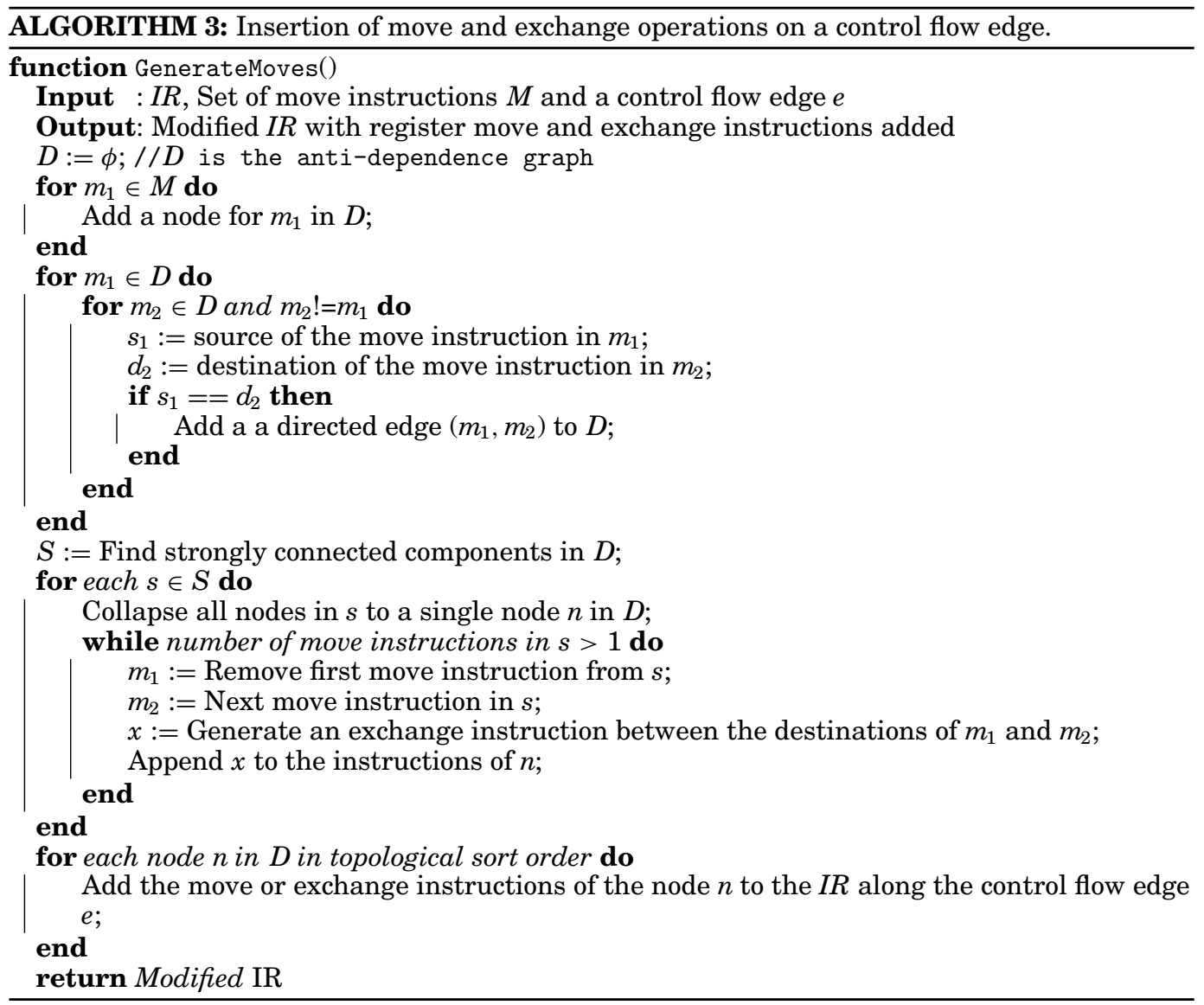

$\mathcal{K}$-that is, it represents all possible anti-dependences between all possible pairs of physical registers. Hence, the overall space complexity is $\mathcal{O}\left(|\mathcal{E}| *\left(|\mathcal{C}|+|\mathcal{K}|^{2}\right)\right)$.

TheOREm 5.4. Spill-free assignment takes $\mathcal{O}\left(|\mathcal{B}|+\left(|\mathcal{E}| *\left(|\mathcal{C}|+|\mathcal{K}|^{2}\right)\right)\right)$ time.

Proof: Similar in nature to the proof for Theorem 5.3.

\subsection{Assignment with Move Coalescing and Register Moves}

Move coalescing is an important optimization in register allocation algorithms that assigns the same physical registers to the source and destination of an IR move instruction when possible to do so. The register assignment phase must try to coalesce as many moves as possible so as to get rid of the move instructions from the $I R$. As we saw in the preceding section, additional register moves may be inserted in the assignment phase instead of spilling. Note that move coalescing approaches using aggressive [Chaitin et al. 1981], conservative [Briggs et al. 1994], and optimistic [Park and Moon 1998] techniques are shown to be NP-complete by Bouchez et al. [2007]. In this section, we first present a coalesce graph (CG) that models both the $I R$ move instructions and register-to-register moves. Then, the register assignment phase on the CG is formulated as an optimization problem that tries to maximize the number of move instructions removed after assignment. We provide a greedy heuristic to solve it. 
Definition 5.5. A CG is an undirected weighted graph $G=\left\langle V, E_{m} \cup E_{r}\right\rangle$, where $V$ represents the basic intervals in $\mathcal{B}$ and an edge $e \subseteq V \times V$ corresponds to the following two types of move instructions between a pair of basic intervals:

(1) $E_{m}$ : the move instructions already present in the $I R$. The weight of such an edge $\mathcal{W}(e)$ is the estimated frequency of the corresponding move instruction.

(2) $E_{r}$ : the move instructions that need to be added on control flow edges for which the two interval end points have different register assignments for the same compound interval. The weight of such an edge $\mathcal{W}(e)$ is the estimated frequency of the control flow edge on which the move instruction is added.

Definition 5.6. Assignment Optimization Problem: Given a set of basic intervals $b \in \mathcal{B}$ with spilled $(b)=$ false, $\mathrm{CG}=\left\langle V, E=\left\{E_{m} \cup E_{r}\right\}\right\rangle, I R$, and $k$ uniform physical registers, find register assignment $\operatorname{reg}(b)$ for every basic interval $b$ such that the following objective function is minimized:

$$
\sum_{\forall e \in E, \quad e=\left(b_{1}, b_{2}\right) \wedge r e g\left(b_{1}\right) !=r e g\left(b_{2}\right)} \mathcal{W}(e)
$$

The assignment guides which additional register-to-register copy or exchange instructions need to be inserted in the $I R$.

Algorithm 4 presents a greedy heuristic to select a physical register for a basic interval $b$ given the $\mathrm{CG}$ and the available set of physical register avail. avail is updated as basic intervals expire. Map is a data structure that maps a physical register to a cost. Steps 3 through 7 find the physical registers and their associated costs that are already assigned to the neighbors of $b$ in the CG (similar to the idea of biased coloring [Briggs et al. 1992]). Our approach takes into account the edges in $E_{r}$ due to register-to-register moves. The greedy heuristic chooses a physical register reg $(b)$ with maximum cost-that is, the benefit of assigning the physical register to basic interval $b$.

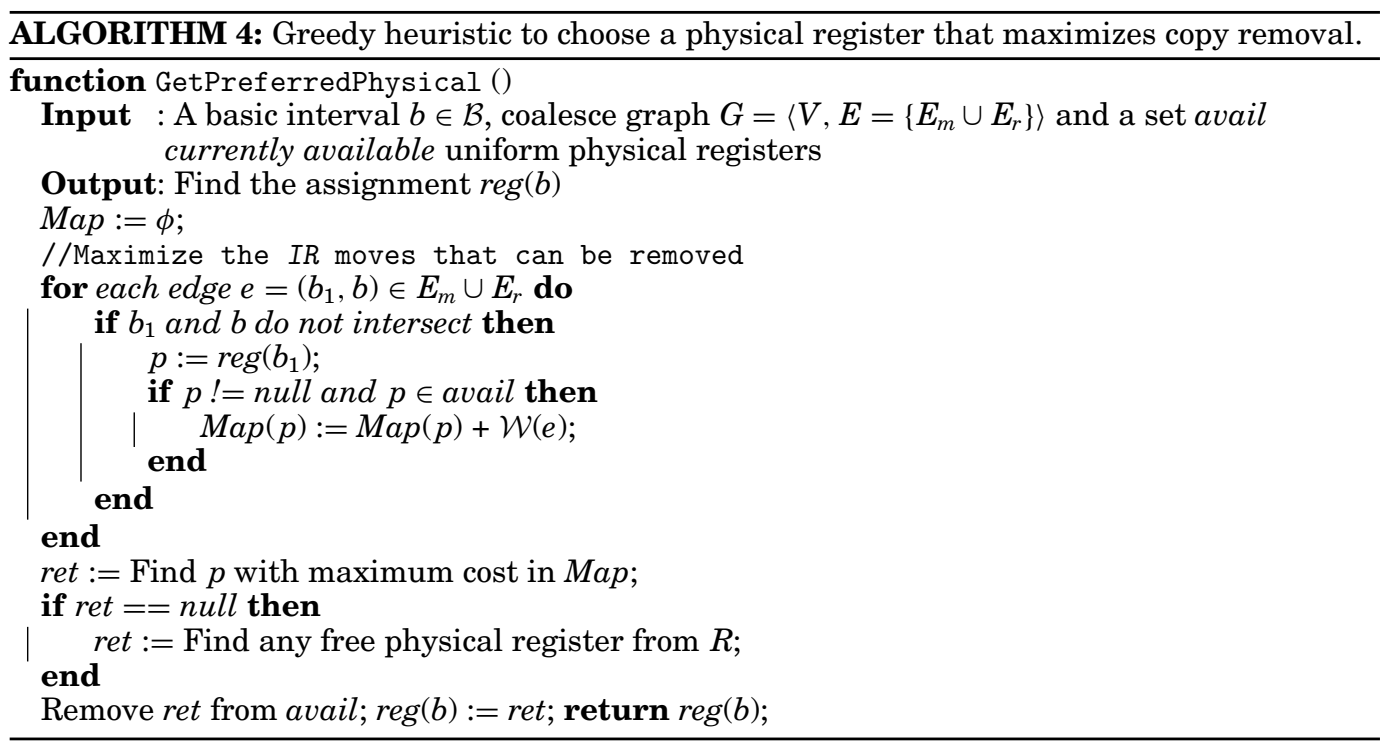


Theorem 5.7. Register assignment using Algorithm 4 requires $\mathcal{O}(|\mathcal{B}|+|\mathrm{IR}|+(|\mathcal{C}| *$ $\left.\left.\max _{c}\right)\right)$ space, where max $_{c}$ denotes the maximum number of basic intervals in a compound interval.

Proof: The additional space requirement is due to the CG containing $|\mathcal{B}|$ number of nodes. $E_{m}$ in the worst case ends up creating $|I R|$ edges. $E_{r}$ adds edges between basic intervals of the same compound interval and hence needs $|\mathcal{C}| * \max _{c}$ number of edges.

THEOREM 5.8. Register assignment using Algorithm 4 takes $\mathcal{O}\left(\left(|\mathcal{B}| * \max _{c}\right)+|\mathrm{IR}|+\right.$ $\left.\left(|\mathcal{E}| *\left(|\mathcal{C}|+|\mathcal{K}|^{2}\right)\right)\right)$ time.

PRoof: In addition to Theorem 5.4, before deciding a physical register for each basic interval $b$, it is required to traverse each of the neighbors in CG. For all basic intervals, this adds overall $2 *|I R|$ time complexity for $I R$ move instructions and $|\mathcal{B}| * \max _{c}$ time complexity for $E_{r}$ edges in CG.

\section{SPILLING AND ASSIGNMENT WITH REGISTER CLASSES}

In the preceding sections, we have described register spilling and assignment for $k$ physical registers that are uniform-that is, they are independent and interchangeable [Smith et al. 2004]. However, modern systems such as x86, HP RA-RISC, Sun SPARC, and MIPS come with physical registers that may not necessarily be interchangeable. For example, the Intel 32-bit x86 architecture provides eight integer physical registers, of which six are typically exposed for register allocation. These six physical registers are further divided into four high-level overlapping register classes based on calling conventions and 8-bit operand accesses. Since the register classes may not necessarily be disjoint, a register allocator must take into account register classes during spilling and assignment to produce high-quality machine code. In this section, we describe how spilling and assignment can be performed in the presence of register classes. We assume calling convention-related constraints are also expressed in additional register classes with infinite spill cost.

\subsection{Constrained Spilling Using BLG}

Allocation in the presence of register classes can be achieved using the following two approaches:

(1) Build BLG for each register class and apply the algorithm in Figure 1 to each BLG in a particular order starting with the most constrained register class that has fewer physical registers in a class. For example, in the 32-bit x86 architecture, we need to build four BLGs for four integer register classes and apply the algorithm in Figure 1 in the order 8-bit nonvolatile (EBX), nonvolatile (EBX, EBP, and EDI), 8-bit volatile (EAX, EBX, ECX, and EDX), and then for the complete integer register class. If a compound interval is spilled in a BLG for a register class, that decision needs to be propagated to the other BLGs of other classes.

(2) An alternative approach is to build a single BLG. During every visit of an interval end point in Figure 1, we make it unconstrained with respect to all other register classes before another end point is visited. This approach is space efficient, as it builds only one BLG but can eagerly generate more spills than (1).

Our experimental results in Section 7 were obtained using Approach (1).

\subsection{Constrained Assignment and Move Coalescing}

Given a CG (as defined in Section 5), when we try to find an assignment for a basic interval $b$, the register classes of the neighbors of $b$ in the CG along with the register class of b play a key role in selecting a physical register for $b$. An IR move instruction 
can be coalesced if source and destination basic intervals have a non-null intersection in their register classes.

Another key point in register assignment is that we no longer can rely on the increasing start point order for assignment of basic intervals, since an early decision of physical register assignment of a register class may result in more symbolic registers being spilled later on or giving up other opportunities for coalescing. We define the register assignment problem in the presence of register classes as an optimization problem that may incur additional spills.

Definition 6.1. Constrained Assignment Optimization Problem: Given a set of basic intervals $b \in \mathcal{B}$ with spilled $(b)=$ false, regclass $(b)$ indicating physical registers that can be assigned to each $b, C G=\left\langle V, E=\left\{E_{m} \cup E_{r}\right\}\right\rangle$, and $I R$, find a register assignment $r e g(b)$ for a subset of basic intervals $S \subseteq \mathcal{B}$ such that the following objective function is minimized:

$$
\sum_{\forall b \in \mathcal{B}-S} S P I L L(b)+\sum_{\forall e \in E,} \sum_{e=\left(b_{1}, b_{2}\right) \wedge \operatorname{reg}\left(b_{1}\right) !=\operatorname{reg}\left(b_{2}\right)} \mathcal{W}(e)
$$

Insert additional register-to-register copy or exchange instructions in the $I R$.

Algorithm 5 presents a bucket-based approach to register assignment that tries to strike a balance between register classes and spill cost. The assignOrder data structure holds sorted basic intervals according to register classes in a two-dimensional array. Each register class is represented as a unique integer id. Steps 2 through 4 compute the total number of basic intervals per register class. Steps 5 through 7 compute the number of elements per bucket. Steps 8 through 13 decide the appropriate bucket in assignOrder where a basic interval should reside (based on next availability). Steps 14 through 17 find an assignment for basic intervals by traversing the assignOrder array in a row major order. The heuristic for assigning a physical register to a basic interval follows a similar approach described in Section 5, except additional care must be taken to account for register class constraints. The details are provided in Algorithm 6.

\section{EXPERIMENTAL RESULTS}

We present an experimental evaluation of the BLG register spilling and assignment algorithms presented in this article. The experimental setup consists of two compiler infrastructures: a static compiler evaluation using LLVM 2.7 [Lettner 2009] and a dynamic compiler evaluation Jikes RVM 3.1.1 [2011]. In the static compilation evaluation, we perform both compile-time and run-time comparisons of our BLG allocator compared to an existing GC implementation [Cooper and Dasgupta 2006] and the LLVM LS [Lettner 2009]. In the dynamic compilation evaluation, we compare our BLG allocator performance compared to the Jikes RVM LS algorithm.

\subsection{LLVM 2.7 Evaluation}

The LLVM evaluations were performed on an Intel Xeon 2.66GHz system with $8 \mathrm{~GB}$ of memory and running RedHat Linux (RHEL 5).

Benchmarks: We used 10 benchmarks from the SPEC CPU2006 benchmark suite [Standard Performation Corporation 2006]. The integer benchmarks used are 401. bzip2, 429.mcf, 458.s jeng, 464.h264ref, and 473. astar. The floating-point benchmarks used are 410.bwaves, 434.zeusmp, 435.gromacs, 444.namd, and 470.1bm. All benchmarks were executed under the optimization level -O2 of LLVM. Since we invoked LLVM in static compilation mode, we ran each benchmark five times and reported the best of the five runs as the runtime performance measurement.

Comparison approaches: Experimental results are reported for the following cases: 


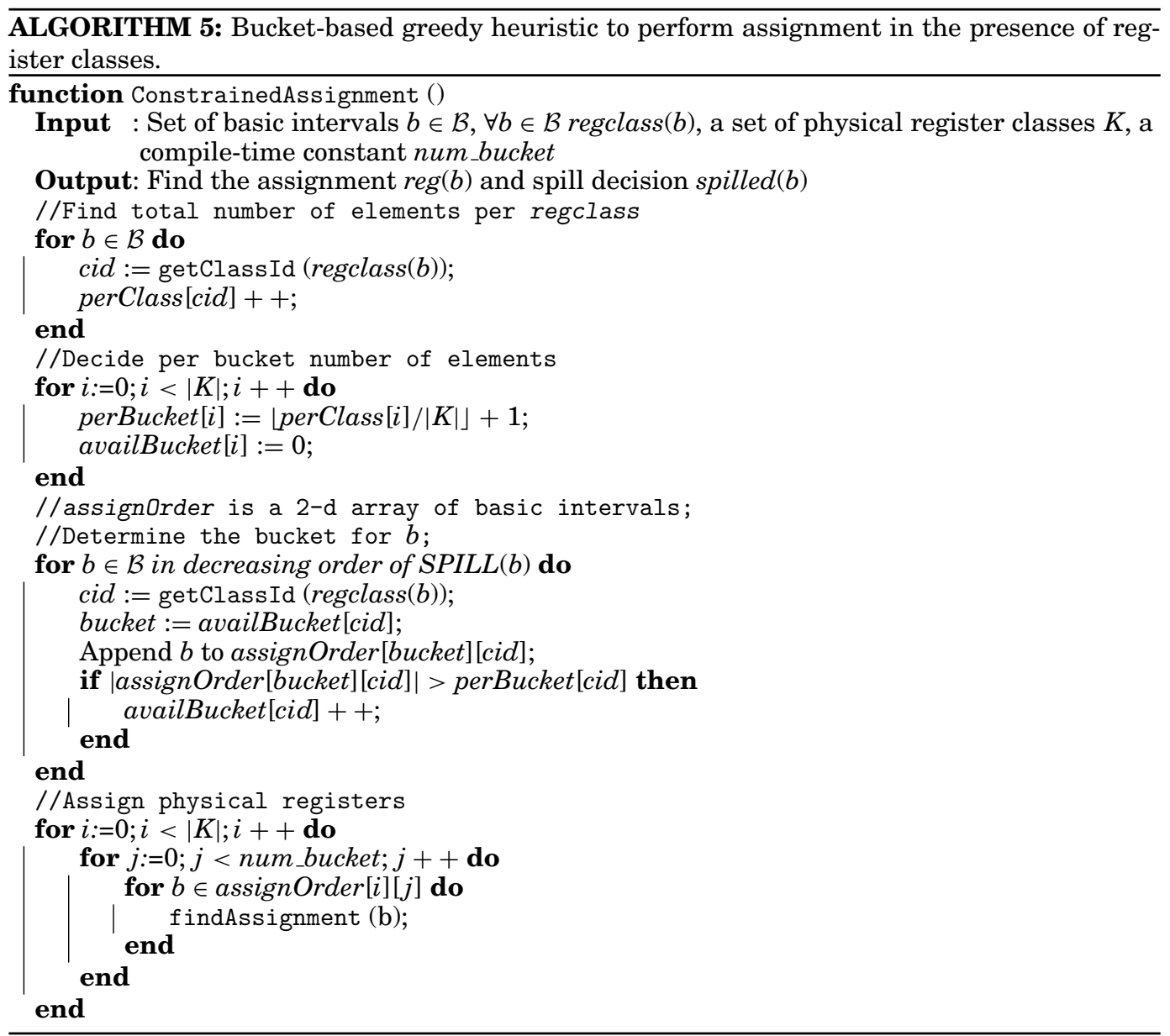

(1) LLVMLS: Baseline measurement using the default LS register allocator in LLVM. This allocator implements aggressive live-range splitting and differs from the standard LS algorithm Poletto and Sarkar [1999] by introducing backtracking. These extensions are described in Wimmer and Mössenböck [2005]. This algorithm also performs aggressive coalescing prior to register allocation.

(2) GC: The Chaitin-Briggs Chaitin et al. [1981] and Briggs et al. [1994] register allocator. This implementation uses the same code base of Chaitin-Briggs allocator with aggressive coalescing that was used in Cooper and Dasgupta [2006]. Details of the Chaitin-Briggs allocator can be found in Briggs et al. [1994].

(3) BLG+LS: The register spilling and assignment algorithm presented in Section 6 with the spill code generation algorithm from (1) - that is, after the allocation and assignment passes are completed using BLG, the $I R$ is rewritten using the physical registers for the nonspilled variables and move code is inserted. The $I R$ is then passed to the LS register allocator of LLVM to generate the spill code. ${ }^{3}$

\footnotetext{
${ }^{3}$ We do not devise any new spill code generation technique-our focus is on spilling and assignment, and thus we use existing spill code generation techniques such as basic block-based spill code generation in LLVM LS and global spill code generation in GC.
} 


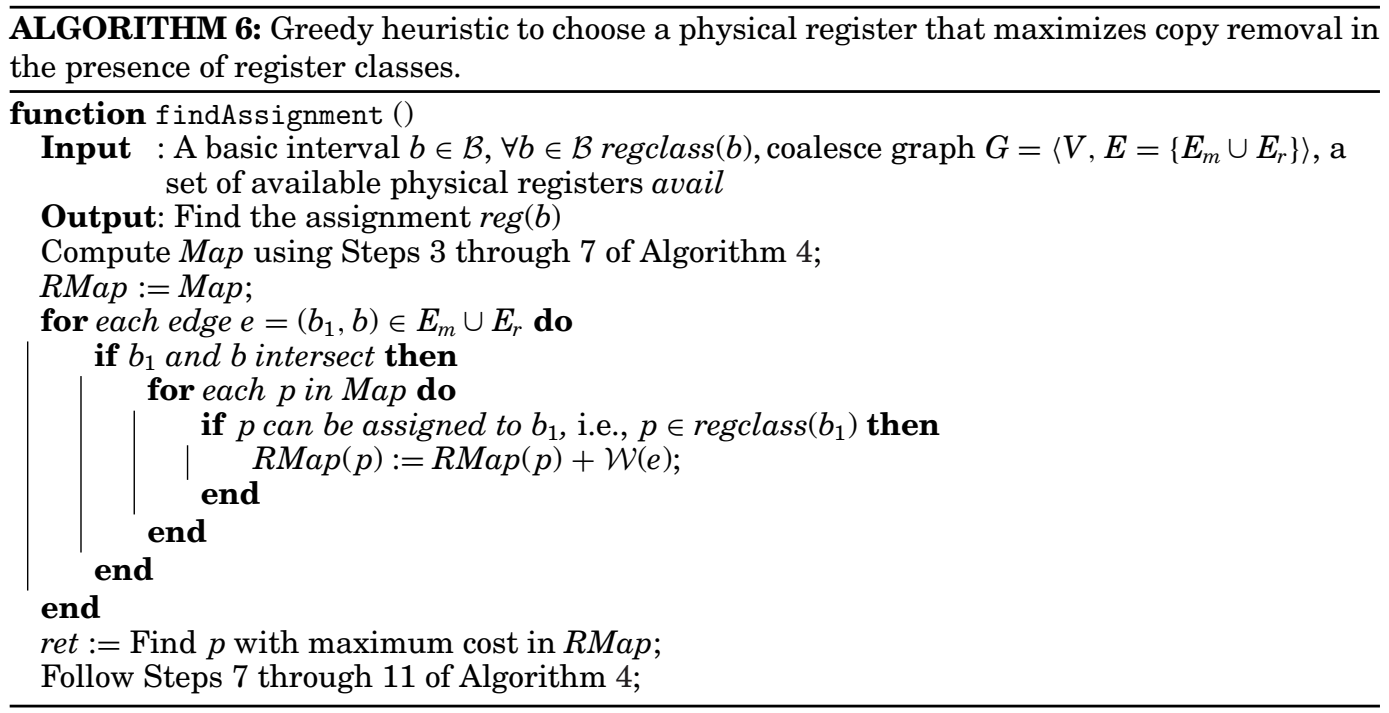

(4) BLG+GS: The register spilling and assignment algorithm presented in Section 6 with the spill code generation algorithm from (2)-that is, after allocation and assignment are completed using BLG, the $I R$ is rewritten using the physical registers for the nonspilled variables and move code is inserted. The $I R$ is then passed to the Chaitin-Briggs register allocator to generate spill code. For the BLG allocator, we set the compile-time constant num_bucket to 5. Note that this approach does not yet implement partial spills.

Compile-Time Comparison: Table I compares the compile-time overheads of BLG versus GC. The measurements were obtained for functions with the largest IGs (in term of number of nodes) in the SPEC CPU2006 benchmarks. Column 3 reports the total number of LLVM IR instructions for the max function. Columns 4 and 5 report the total number of nodes and edges in the IG, respectively. (We only report these numbers for the first iteration of the Chaitin-Briggs allocator-subsequent iterations require additional smaller IGs.) Columns 5 and 6 report the total number of nodes and edges in BLG that only considers constrained interval end points (i.e., those end points with MAXLIVE $>k$; unconstrained interval end points are not necessary, as described in Section 4). We define the Space Usage Ratio metric as the ratio of the following two quantities: (1) sum of columns 3 through 5 (|IG|) and (2) sum of columns 3,6 , and 7 (BLG). This metric varies from $2.9 \times$ to $13.7 \times$ in our case, indicating the lower space usage of BLG compared to GC. Whereas theoretically both IG and BLG can be quadratic, in practice, we observe BLG to be much smaller than IG.

Runtime Comparison: Figure 3 reports the relative performance improvement of the register allocation algorithm presented in this article along with Chaitin-Briggs spill code generator, BLG+GS, compared to the original Chaitin-Briggs allocatorthat is, GC on the Intel Xeon system. We observe a performance improvement of up to $7.87 \%$ in 464. h264ref benchmark, and we do not observe any degradation in any of the benchmarks. While comparing our BLG allocator with LS spill code generator (i.e., BLG+LS) with that of LLVM's default register allocator LLVM+LS (as shown in Table II), we did not observe any noticeable performance difference. The reason is that the default LLVM register allocator implements other register allocation techniques, 
Table I. Comparison of Compile-Time Statistics Between BLG+LS and GC for SPEC CPU2006 Benchmarks

\begin{tabular}{|c|c|c|c|c|c|c|c|c|c|}
\hline Benchmark & $\begin{array}{c}\text { Max } \\
\text { Function }\end{array}$ & $|\mathrm{IR}|$ & $\begin{array}{c}\mathrm{IG} \\
\text { \#nodes }\end{array}$ & $\begin{array}{c}\mathrm{IG} \\
\text { \#edges }\end{array}$ & $\begin{array}{c}\text { BLG } \\
\text { \#nodes }\end{array}$ & $\begin{array}{c}\text { BLG } \\
\text { \#edges }\end{array}$ & $\begin{array}{l}\text { Space } \\
\text { Usage } \\
\text { Ratio }\end{array}$ & $\begin{array}{c}\text { BLG } \\
\text { \#nodes } \\
\text { opt }\end{array}$ & $\begin{array}{c}\text { BLG } \\
\text { \#edges } \\
\text { opt }\end{array}$ \\
\hline 401.bzip2 & sendMTFValues & 3545 & 2,693 & 53,562 & 1,844 & 9,819 & 3.9 & 1,721 & 8,823 \\
\hline 410.bwaves & $\begin{array}{l}\text { bi_cgstab_ } \\
\text { block_ }\end{array}$ & 2083 & 1,025 & 5,430 & 134 & 269 & 3.4 & 134 & 269 \\
\hline 429.mcf & read_min & 440 & 279 & 3,376 & 47 & 49 & 7.6 & 47 & 49 \\
\hline 434.zeusmp & setup_ & 5147 & 3,030 & 33,138 & 387 & 1,750 & 5.6 & 79 & 210 \\
\hline 435.gromacs & do_inputrec & 3519 & 1,941 & 36,606 & 64 & 142 & 11.3 & 39 & 67 \\
\hline 444.namd & $\begin{array}{l}\text { ZN20Compute- } \\
\text { NonbondedUtil- } \\
\text { 30calc_self- } \\
\text { _energy_- } \\
\text { fullelect_fep- } \\
\text { EP9nonbonded }\end{array}$ & 2244 & 907 & 6,156 & 4 & 3 & 4.1 & 4 & 3 \\
\hline 458.sjeng & std_eval & 1316 & 812 & 7,908 & 0 & 0 & 7.63 & 0 & 0 \\
\hline 464.h264ref & $\begin{array}{l}\text { SubPelBlock- } \\
\text { SearchBiPred }\end{array}$ & 5787 & 4,757 & 86,092 & 356 & 921 & 13.7 & 53 & 55 \\
\hline $470.1 \mathrm{bm}$ & $\begin{array}{l}\text { LBM_handleIn- } \\
\text { OutFlow }\end{array}$ & 1162 & 643 & 5,380 & 189 & 270 & 4.4 & 189 & 270 \\
\hline 473.astar & $\begin{array}{l}\text { ZN6wayobj18- } \\
\text { makeobstacle- } \\
\text { bound2EPiiS0_ }\end{array}$ & 382 & 295 & 438 & 0 & 0 & 2.9 & 0 & 0 \\
\hline
\end{tabular}

The number of compound intervals (i.e., variables) for BLG is the same as in column 4. The Space Usage Ratio in column 8 is the ratio of the following two quantities: (1) sum of $|I R|$, IG nodes, and IG edges; (2) $|I R|$, BLG nodes, and BLG edges. Columns 9 and 10 report the BLG nodes and edges after optimizing BLG for space.

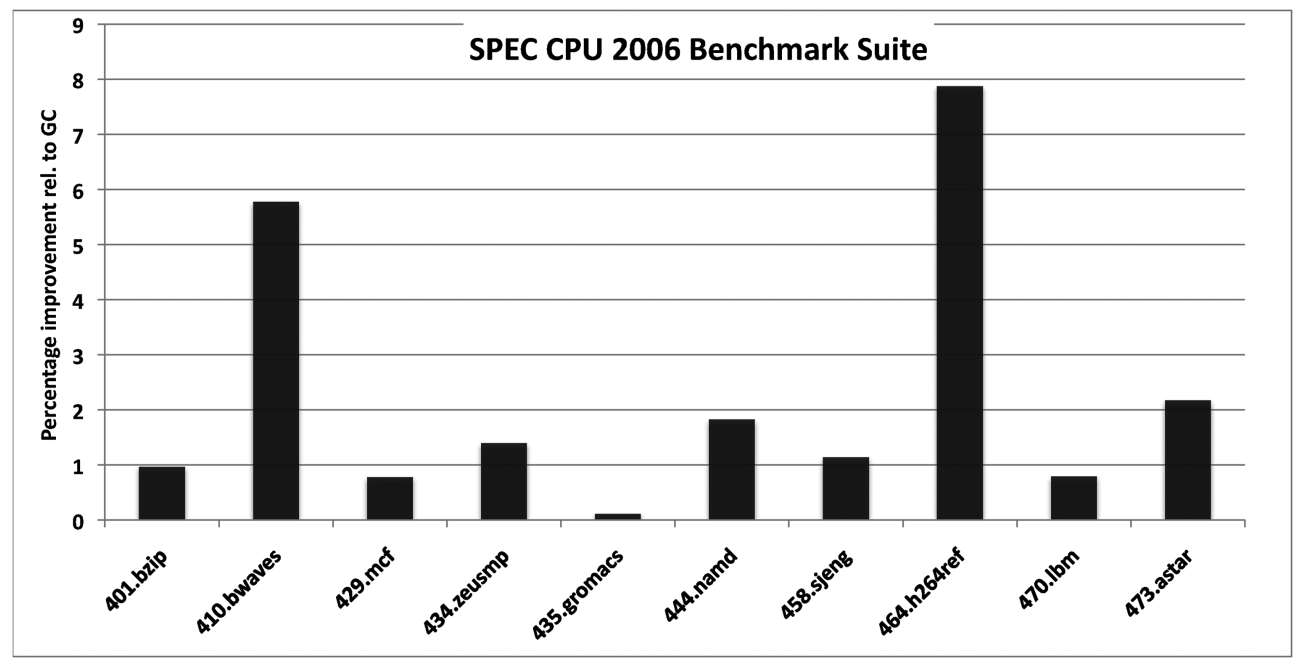

Fig. 3. Percentage improvement of execution times obtained by BLG+GS, (i.e., BLG+Chaitin-Briggs spiller) compared to GC in the LLVM static compiler infrastructure for SPEC CPU2006 benchmarks on the Intel Xeon system. 
Table II. Comparison of Runtime Performance of BLG+LS Versus LLVM LS Register Allocation

\begin{tabular}{|l||c|c|}
\hline \multicolumn{1}{|c||}{ BLG+LS } & LLVM+LS \\
Benchmark & execution time (in sec) & execution time (in sec) \\
\hline 401.bzip & 9.9 & 10.0 \\
410.bwaves & 2856.4 & 2853.1 \\
429.mcf & 6.7 & 6.8 \\
434.zeusmp & 40.4 & 40.5 \\
435.gromacs & 2079.1 & 2076.7 \\
444.namd & 38.1 & 38.1 \\
458.sjeng & 11.0 & 11.1 \\
464.h264ref & 1806.4 & 1806.4 \\
470.lbm & 1.6 & 1.6 \\
473.astar & 23.5 & 23.5 \\
\hline
\end{tabular}

Execution Time: Comparison of execution times obtained by BLG+LS, (i.e., BLG+LS Spiller) compared to the default LLVM LS for SPEC CPU2006 benchmarks using LLVM static compiler on the Intel system. Note that LLVMLS performs additional optimizations, such as live-range splitting and backtracking compared to BLG+LS.

such as aggressive live-range splitting and backtracking, in order to help moderate register pressure during spilling and assignment phases. The ad hoc heuristic via backtracking in LLVM performs unspilling recursively in order to avoid reserved spill registers, and this pass has a quadratic complexity as described in Evlogimenos [2004]. Additionally, our scheme without any sophisticated live-range splitting mechanism is able to match the performance of state-of-the-art LLVM. In the future, we would like to devise live-range splitting heuristics for BLG that exploit the structure of the program [Lueh et al. 2000; Appel and George 2001].

\subsection{Jikes RVM 3.1.1 Evaluation}

The Jikes RVM evaluations were performed on two systems: (1) Intel Xeon $2.66 \mathrm{GHz}$ system with 8GB of memory and running RedHat Linux (RHEL 5); (2) PowerPC 7 2.66GHz system with 8GB memory, running SUSE Linux.

Benchmarks: We used the serial benchmarks in v2.0 of the Java Grande Forum (JGF) benchmark suite [ECCC 2001] and the Dacapo 2006 benchmark suite [Blackburn et al. 2006] to evaluate the performance of our register allocator. We choose the five large benchmarks from Section 3 (raytracer, moldyn, montecarlo, euler, and search). ${ }^{4}$ For the Dacapo benchmark suite, we report performance evaluation of 10 benchmarks out of a total of 11 benchmarks. These include antlr, bloat, fop, hsqldb, jython, luindex, pmd, xalan, lusearch, and eclipse. ${ }^{5}$ Further, for PowerPC 7 evaluation, we could not compile lusearch and luindex benchmarks in Jikes RVM 3.1.1.

Compiler: The boot image for Jikes RVM used a production configuration. Since the Jikes RVM release did not support generation of Intel exchange instruction, we modified its assembler to add this support. Jikes RVM uses SSE registers for storing double/floating point values. However, to the best of our knowledge, a direct exchange instruction to swap values in SSE registers does not exist, so we generate three xor instructions to exchange a pair of float/double values. The exchange instructions are generated judiciously - that is, if there is a free physical register available for swapping the values, an exchange instruction is not generated [Boissinot et al. 2009]. For all Java runs, the execution times are reported for dynamic compilation (both runtime

\footnotetext{
${ }^{4}$ Results for Section 1 and 2 benchmarks have been omitted because they are smaller benchmarks.

${ }^{5}$ chart is omitted, as it requires a special AWT library to compile, and the existing Jike RVM is unable to compile it.
} 


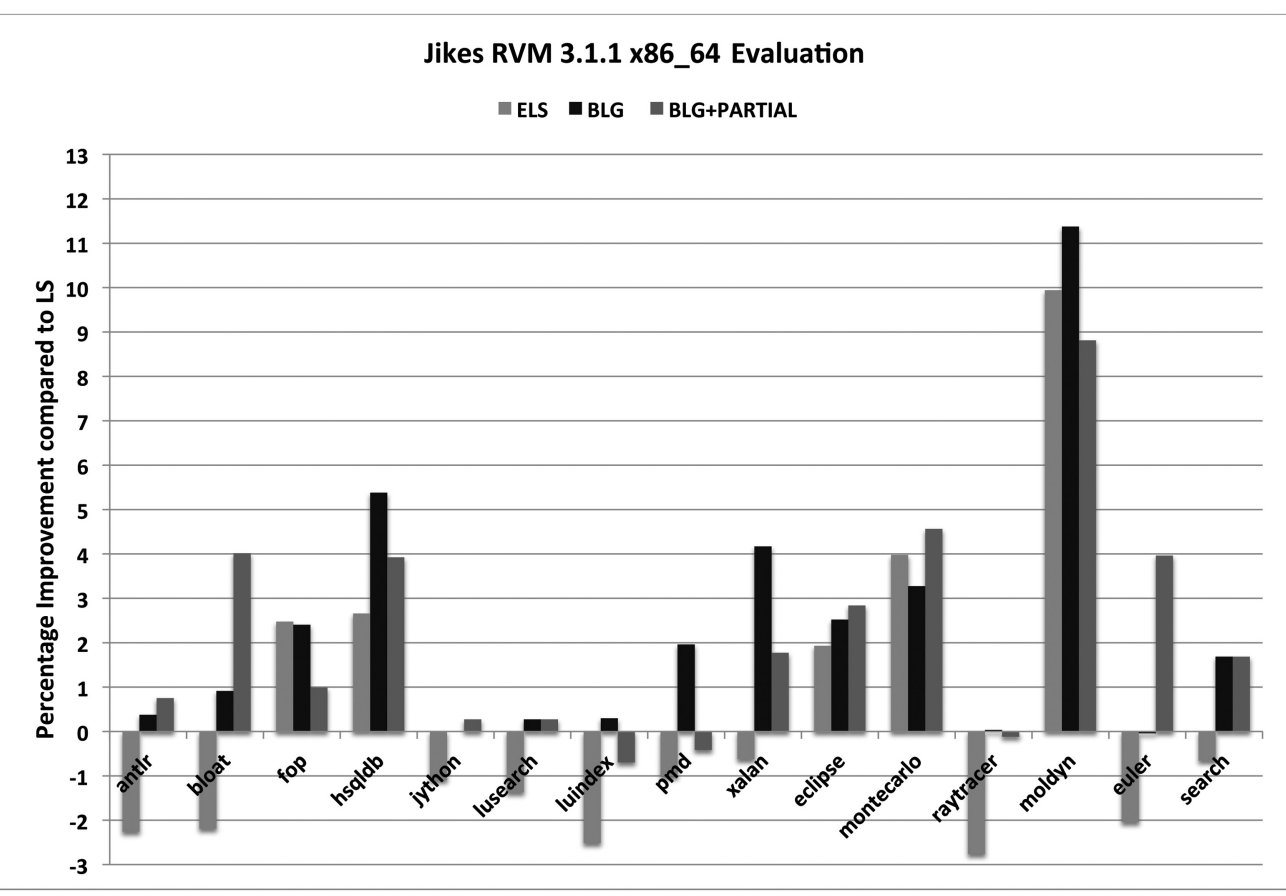

Fig. 4. Percentage improvement of ELS, BLG, and BLG+PARTIAL compared to LS in Jikes RVM dynamic compiler on a $\times 86 \_64$ Intel Xeon system.

and compile time) and use the methodology described in Georges et al. [2007] - that is, we report the average runtime performance of 30 runs within a single VM invocation along with the execution variance that uses a $95 \%$ confidence interval.

Comparison Approaches: Experimental results in Jikes RVM evaluation are reported for the following cases: (1) LS: Baseline measurement with LS register allocator in Jikes RVM that uses the algorithm from Poletto and Sarkar [1999] with extensions for live-range "holes"; (2) ELS: The ELS algorithm from Sarkar and Barik [2007]; (3) BLG: The BLG register allocation algorithm presented in Section 6; and (4) BLG+PARTIAL: The BLG register allocation algorithm with partial spills presented in Section 4.1. The compile-time constant num_bucket in Figure 5 is set to 5 for all runs. Increasing this number to a higher value does not obviously impact the runtime performance.

Runtime Comparison: Figure 4 reports the relative performance improvements for ELS, BLG, and BLG+PARTIAL allocators compared to the default LS allocator of Jikes RVM on the Intel Xeon system. The BLG register allocator resulted in a performance improvement in the range of $-0.04 \%$ to $11.37 \%$ (for moldyn). The BLG+PARTIAL register allocator resulted in a performance improvement in the range of $-0.69 \%$ to $8.81 \%$ (for moldyn). For the moldyn benchmark, the most frequently executed function is force. MAXLIVE for this function is $>7$. (Jikes RVM uses 8 SSE registers for storing double/float values, and one out of them, XMM7, is used for scratch register.) Spilling decisions for this method impact the performance of the benchmark significantly. BLG for this method coalesces more moves than LS and is able to spill 14 symbolic registers compared to 16 symbolic registers in LS. The BLG+PARTIAL allocator improves performance for bloat, eclipse, montecarlo, and euler benchmarks when compared to BLG. The runtime performance benefits for both BLG and BLG+PARTIAL are not 


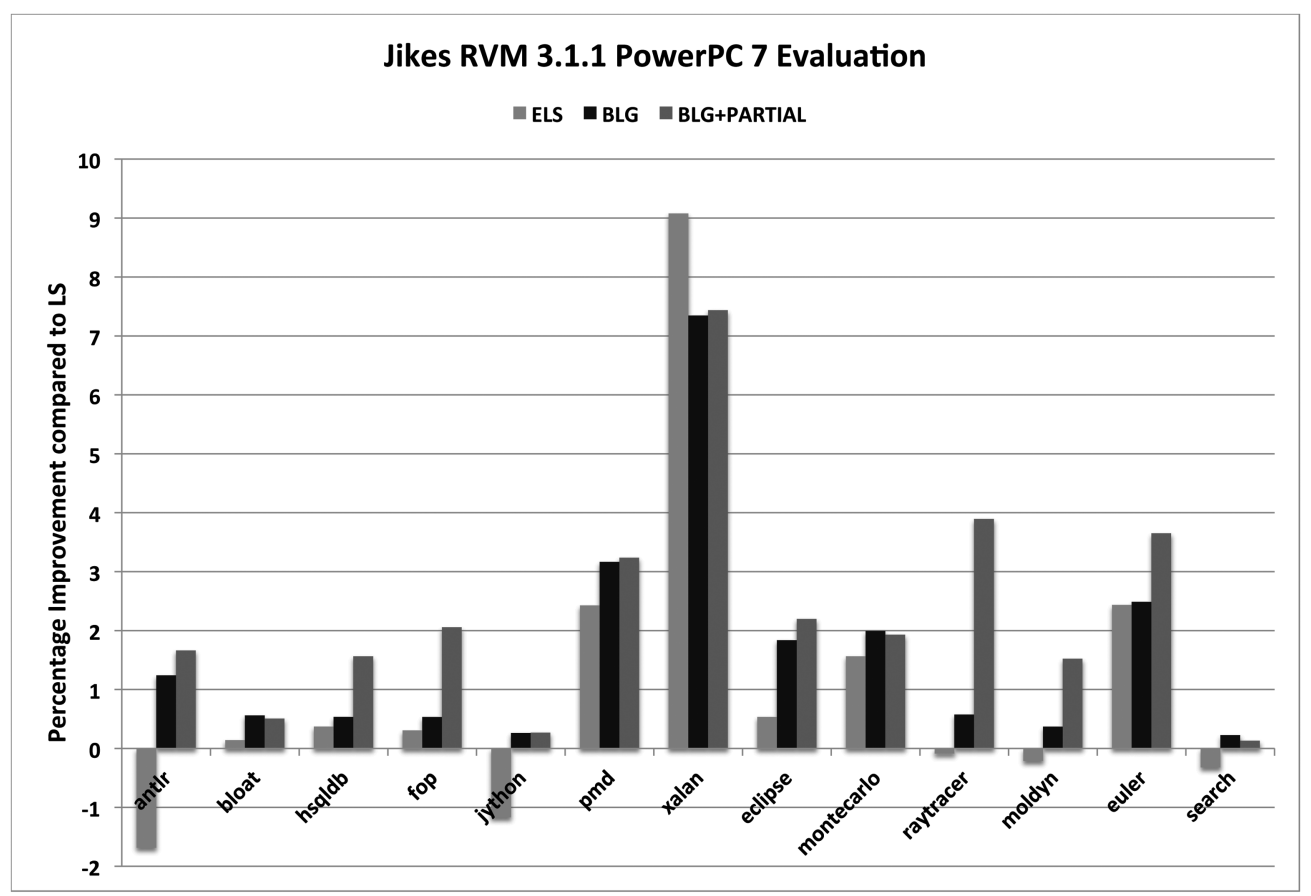

Fig. 5. Percentage improvement of ELS, BLG, and BLG+PARTIAL compared to LS in Jikes RVM dynamic compiler on a PowerPC 7 system.

surprising, as they perform global spill decisions on a BLG compared to the local spill decisions made by LS and ELS. We observed a slowdown of $0.69 \%, 0.41 \%$, and $0.12 \%$ for luindex, pmd, and raytracer for BLG+PARTIAL: our current heuristic splits live ranges only at basic interval granularity, which may not be optimal. More sophisticated live-range splitting is left for future work.

On the PowerPC 7 system, we observe performance improvements for BLG allocator compared to LS in the range of $0.23 \%$ to $7.34 \%$ (for xalan), as shown in Figure 5. The BLG+PARTIAL allocator is able to improve performance for most of the benchmarks.

Compile-Time Comparison: Table III reports compile-time comparison of BLG versus LS. As described in previous sections, LS is best for compile-time efficiency, as it performs both spilling and assignment in just one pass over the basic intervals. BLG adds extra new passes for spilling and unspilling via bipartite graph Section 4, move-code generation Section 5, and move coalescing optimization Section 5.2. Thus, BLG is expected to perform slower than LS. We observe an increase in compile time from $2.02 \times$ to $5.37 \times$ for BLG versus LS. This increase in compile time is insignificant compared to the total execution time of a benchmark, since BLG outperforms LS for all benchmarks except euler on the Xeon system and for all benchmarks on the PowerPC 7 system. Interestingly, in our current implementation, we observe that the move-code generation component consumes maximum time. This is because it may require the construction of a move graph and performs SCC search in this graph for a control flow edge. In the future, we would like to optimize the compile time of this phase.

Static Spill-Cost Savings: Figure 6 reports the percentage improvement in static spill cost for BLG compare to LS. The static frequency estimates are computed using standard technique where a spill instruction inside a loop is estimated as $10^{d}$, where $d$ denotes loop depth. We observe reduction in static spill cost for all workloads. For 
Table III. Jikes RVM: Compile-Time Comparison of BLG Versus LS in Jikes RVM Dynamic Optimizing Compiler

\begin{tabular}{|l||c|c|c|}
\hline Benchmark & $\begin{array}{c}\text { BLG } \\
\text { Comp. } \\
\text { Time } \\
(\mathrm{ms})\end{array}$ & $\begin{array}{c}\text { LS } \\
\text { Comp. } \\
\text { Time } \\
(\mathrm{ms})\end{array}$ & $\begin{array}{c}\text { Relative } \\
\text { Increase } \\
\text { in Comp. } \\
\text { Time (BLG/LS) }\end{array}$ \\
\hline antlr & 0.35 & 0.07 & 5.37 \\
bloat & 1.43 & 0.3 & 4.76 \\
fop & 0.08 & 0.03 & 2.44 \\
hsqldb & 0.87 & 0.28 & 3.08 \\
jython & 0.6 & 0.11 & 5.27 \\
luindex & 1.08 & 0.22 & 4.8 \\
pmd & 0.78 & 0.21 & 3.62 \\
xalan & 0.9 & 0.31 & 2.89 \\
eclipse & 1.58 & 0.78 & 2.02 \\
montecarlo & 0.09 & 0.02 & 3.8 \\
raytracer & 0.05 & 0.02 & 3.23 \\
moldyn & 0.02 & 0.01 & 2.83 \\
euler & 0.26 & 0.1 & 2.5 \\
search & 0.05 & 0.015 & 3.2 \\
\hline \multicolumn{2}{|r}{}
\end{tabular}

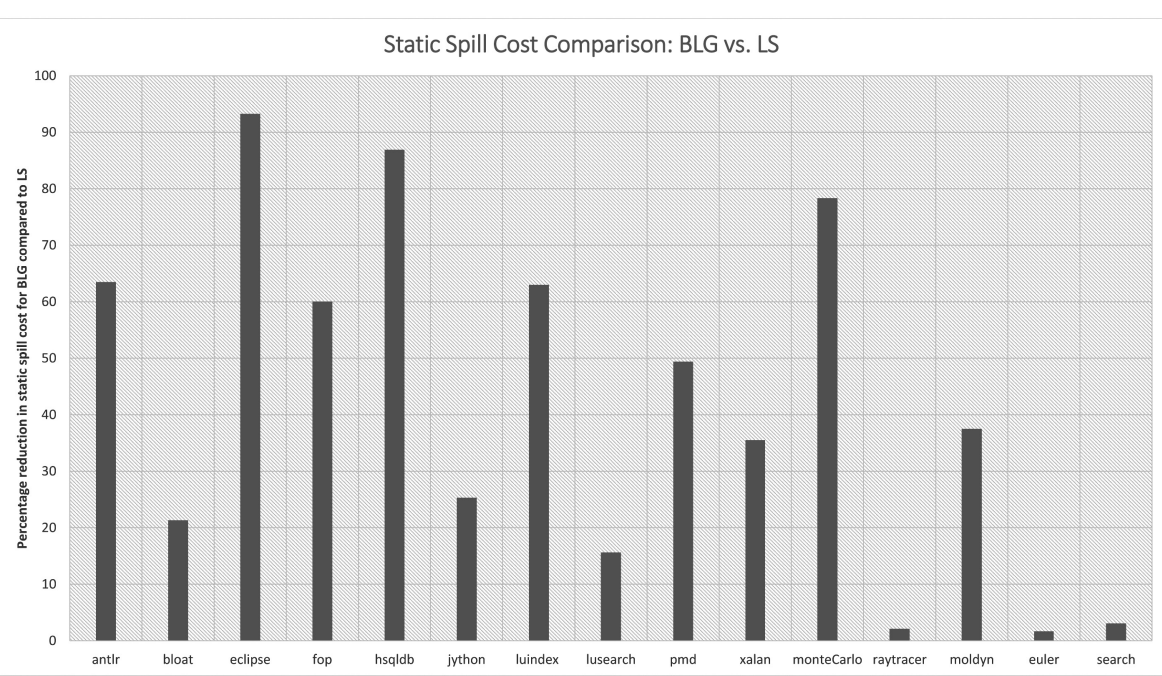

Fig. 6. Percentage reduction in static spill cost for BLG compared to LS in Jikes RVM dynamic compiler.

eclipse, we reduce the spill cost by $93 \%$, which is significant. Please keep in mind that these static measures may not directly correlate to runtime performances due to pipelining and caching effects.

\section{RELATED WORK}

Spill-free register allocation of general programs is NP-complete [Chaitin et al. 1981]. There exists a plethora of past works in using GC-based approaches to spill-free register allocation [Chaitin et al. 1981; Briggs et al. 1989, 1994; Park and Moon 1998; George and Appel 1996; Budimlic et al. 2002; Callahan and Koblenz 1991; Gupta et al. 1994; Smith et al. 2004; Cooper and Dasgupta 2006]. The key data structures of a GC-based algorithm are live ranges and the IG. Allocation phase is performed on the IG by removing the live ranges of degree fewer than $k$. In cases where every live range has 
degree more than or equal to $k$, a live range having lowest spill cost is chosen for spilling. The live ranges that are removed from the IG are assigned physical registers based on the reverse order in which the live ranges were removed from the IG. One of the key limitations of GC-based register allocation is that the live ranges introduce imprecision that may lead to making the IG uncolorable (like the one seen in Figure 3). In contrast, our approach builds on the simple foundations of LS register allocation like intervals and precisely captures liveness information using a novel BLG data structure, which is used for spill-free register allocation [Sarkar and Barik 2007].

Recently, the focus in GC-based register allocation has shifted to SSA-based register allocation [Hack and Goos 2006; Brisk et al. 2005; Brisk 2006; Colombet et al. 2011; Bouchez 2009; Pereira and Palsberg 2005, 2009; Braun et al. 2010]. In SSA representation, the IG is chordal and can be colored optimally in linear time. Like our approach and others in the literature [Appel and George 2001], current approaches to SSA register allocation separate between allocation and assignment phases in register allocation. However, an SSA register allocation incurs additional complexity of dealing with parallel-copy statements during out-of-SSA translation [Hack and Goos 2008; Brisk 2006] and also of dealing with repairing [Colombet et al. 2011]. Our BLG allocator does not need an IG for allocation and efficiently inserts a few register-toregister moves and exchange operations during assignment as opposed to expensive approaches to eliminate a large number of parallel-copy instructions in SSA-based register allocation.

LS [Poletto and Sarkar 1999; Traub et al. 1998; Wimmer and Mössenböck 2005; Thammanur and Pande 2004; Wimmer and Franz 2010; Sarkar and Barik 2007] register allocation algorithms have been preferred for JIT-compilers such as Jikes [2011], HotSpot [Kotzmann et al. 2008], and LLVM [Lattner 2009] due to their low compilationtime and space complexity. Compared to existing LS algorithms, our approach separates allocation and assignment phases. This leads to a much better global spilling decision using a novel bipartite graph. Traditional LS algorithms often combine allocation and assignment for efficiency reasons and hence end up making local spill decisions that lead to performance lag. In the spill-free register allocation algorithm presented in the ELS algorithm [Sarkar and Barik 2007], the spill decisions are taken locally at every program point (i.e., each interval end point is eagerly made completely unconstrained before moving onto another). This is the reason why they had observed a slowdown in SPEC benchmark 181 . mcf. In contrast, the BLG-based allocation algorithm described in this article makes global decisions using the BLG data structure that decides the symbolic registers that need to be spilled to keep the overall spill cost minimized. Additionally, this article describes move coalescing optimizations (in Section 5), register allocation in the presence of register classes (in Section 6), and partial spills (in Section 4.1). More recently, a tree-based register allocation algorithm has been proposed in Rong [2009] that imposes a partial ordering among the basic blocks during coloring and assignment phases, unlike the total order imposed in LS.

The GC-based register allocation algorithm was first extended to handle register classes and aliasing by Smith et al. [2004]. The problem of spill-free register allocation is NP-complete even in the presence of register classesand aliasing [Lee et al. 2007]. The approach taken by Smith et al. is to handle register classes and aliasing by exploiting the coloring constraints on each node of the IG. This approach is elegant and can be easily integrated into any GC register allocation algorithm. More recently, a new LS register allocation algorithm based on puzzle solving was introduced by Pereira and Palsberg [2008, 2010] to handle precoloring and aliasing issues in register allocation. Their approach views the register file as a puzzle and the program variables as puzzle pieces. For many common architectures, the register allocation using puzzles can be 
solved in polynomial time. Our BLG register allocator handles these architectural constraints without building the IG. For the allocation phase, we construct BLG for each register class and propagate spill information across BLGs of other register classes. For the assignment phase, we use a bucket-based approach that strikes a balance between spill cost and move code optimization.

A bipartite graph-based register assignment phase was proposed by Zhang et al. [2004] that is performed on hot paths of an already register allocated code- that is, as a postregister allocation pass. The spilled variables on the hot path form one set of vertices of the bipartite graph, whereas the other set of vertices consists of the set of dead physical registers. An edge is added to their bipartite graph if both the spilled variable and dead physical register are alive in the same basic block. The weight of such an edge is the spill cost of the spilled variable in the basic block. Dead register assignment is then performed using weighted bipartite graph matching. This approach differs from our BLG allocator in many ways: (1) the nodes, edges, and weights of the bipartite graph are all different, and (2) our BLG represents liveness information and solves the allocation phase of register allocation.

The meeting graph model for loop cyclic register allocation described in Eisenbeis et al. [1995] is different from the BLG model. The meeting graph captures information about nonoverlapping intervals (i.e., an edge is added when one interval ends and another starts). This information is useful for obtaining bounds for optimal coloring inside loops. In contrast, BLG captures liveness information at high-pressure program points, which is used to perform global register allocation.

\section{CONCLUSIONS}

In this article, we addressed the problem of developing a register allocation algorithm that builds on the simplicity of LS while improving its runtime performance. It does so by separating the spilling and assignment phases. The spilling phase is modeled as an optimization problem on BLGs-a new data structure introduced in this work. In the spilling and assignment phase, we focus on reducing the number of spill instructions by using register-to-register move and exchange instructions wherever possible to maximize the use of registers. We model register assignment as a second optimization problem that includes move coalescing, as well as register class constraints, and provide a heuristic solution to this problem as well. Our implementation of BLG-based register allocation phase combined with the constrained assignment in Jikes RVM demonstrates runtime performances improvements in the range of $-0.04 \%$ to $11.37 \%$ and in the range of $0.23 \%$ to $7.34 \%$ on Intel Xeon and PowerPC 7 systems, respectively. Additionally, we observe a performance improvement of up to $7.87 \%$ for SPEC CPU2006 benchmarks using our BLG register allocator that uses a GC-based spill code generator when compared to the Chaitin-Briggs register allocator on the Intel Xeon system.

These results show that the BLG register allocation algorithm is a promising alternative to the large body of register allocators existing today. Possible directions for future work include support for more aggressive live-range splitting, backtracking, and studying the impact of move and exchange instructions on code size compared to spill load/store instructions. Further, we would like to study the combined effect of BLG with instruction scheduling.

\section{ACKNOWLEDGMENTS}

We would like to thank the anonymous reviewers for their comments and suggestions on the past submissions related to this article. 


\section{REFERENCES}

Appel, A. W. ANd George, L. 2001. Optimal spilling for CISC machines with few registers. In Proceedings of the ACM SIGPLAN 2001 Conference on Programming Language Design and Implementation (PLDI'01). ACM, New York, NY, 243-253.

Bergner, P., Dahl, P., Engebretsen, D., And K., O’Keefe, M. 1997. Spill code minimization via interference region spilling. SIGPLAN Not. 32, 5, 287-295.

Blackburn, S. M., Garner, R., Hoffman, C., Khan, A. M., McKinley, K. S., Bentzur, R., Diwan, A., Feinberg, D., Frampton, D., Guyer, S. Z., Hirzel, M., Hosking, A., Jump, M., Lee, H., Moss, J. E. B., Phansalkar, A., Stefanović, D., VanDrunen, T., von Dincklage, D., and Wiedermann, B. 2006. The DaCapo benchmarks: Java benchmarking development and analysis. In Proceedings of the 21st Annual ACM SIGPLAN Conference on Object-Oriented Programing, Systems, Languages, and Applications (OOPSLA'06). ACM Press, New York, NY, 169-190.

Boissinot, B., Darte, A., Rastello, F., de Dinechin, B. D., and Guillon, C. 2009. Revisiting out-of-SSA translation for correctness, code quality and efficiency. In Proceedings of the 7th Annual IEEE/ACM International Symposium on Code Generation and Optimization (CGO'09). IEEE Computer Society, Washington, DC, 114-125.

Bouchez, F. 2009. A Study of Spilling and Coalescing in Register Allocation as Two Separate Phases. PhD Dissertation.

Bouchez, F., DARTe, A., AND RAstello, F. 2007. On the complexity of register coalescing. In Proceedings of the Annual IEEE/ACM International Symposium on Code Generation and Optimization (CGO'07). IEEE Computer Society, Washington, DC, 102-114.

Braun, M., Mallon, C., AND Hack, S. 2010. Preference-guided register assignment. In Compiler Construction 2010. Lecture Notes in Computer Science, vol. 6011. Springer, 205-223.

Briggs, P., Cooper, K. D., Kennedy, K., And Torczon, L. 1989. Coloring heuristics for register allocation. In Proceedings of the 1989 SIGPLAN Conference on Programming Language Design and Implementation. $24,7,275-284$.

Briggs, P., Cooper, K. D., ANd Torczon, L. 1992. Rematerialization, In Proceedings of the ACM SIGPLAN 1992 Conference on Programming Language Design and Implementation (PLDI'92).

Briggs, P., Cooper, K. D., And Torczon, L. 1994. Improvements to graph coloring register allocation. ACM Transactions on Programming Languages and Systems 16, 3, 428-455.

BRISK, P. 2006. Advances in Static Single Assignment Form and Register Allocation. PhD Dissertation. Los Angeles, CA.

Brisk, P., Dabiri, F., Macbeth, J., And Sarrafzadeh, M. 2005. Polynomial time graph coloring register allocation. In Proceedings of the 14th International Workshop on Logic and Synthesis.

Budimlic, Z., Cooper, K. D., Harvey, T. J., Kennedy, K., Oberg, T. S., and Reeves, S. W. 2002. Fast copy coalescing and live-range identification. In Proceedings of the ACM SIGPLAN 2002 Conference on Programming Language Design and Implementation (PLDI'02). ACM, New York, NY, 25-32.

Callahan, D. And Koblenz, B. 1991. Register allocation via hierarchical graph coloring. In Proceedings of the ACM SIGPLAN 1991 Conference on Programming Language Design and Implementation (PLDI'91). ACM Press, New York, NY, 192-203.

Chaitin, G. J., Auslander, M., Chandra, A., Cocke, J., Hopkins, M., and Markstein, P. 1981. Register allocation via coloring. Computer Languages 6, 47-57.

Colombet, Q., Boissinot, B., BRisk, P., Hack, S., AND RAstello, F. 2011. Graph-coloring and treescan register allocation using repairing. In Proceedings of the 14th International Conference on Compilers, Architectures and Synthesis for Embedded Systems (CASES'11). ACM, New York, NY, 45-54.

Cooper, K. D. AND DASGUPTA, A. 2006. Tailoring graph-coloring register allocation for runtime compilation. In Proceedings of the Annual IEEE / ACM International Symposium on Code Generation and Optimization (CGO'06). IEEE Computer Society, Washington, DC, 39-49.

Eisenbeis, C., Lelait, S., And Marmol, B. 1995. The meeting graph: A new model for loop cyclic register allocation. In Proceedings of the IFIP WG10.3 Working Conference on Parallel Architectures and Compilation Techniques (PACT'95). IFIP Working Group on Algol, Manchester, UK, 264-267.

EPCC. 2001. The Java Grande Forum Benchmark Suite. Retrieved from http://www.epcc.ed.ac.uk/ javagrande/javag.html.

Evlogimenos, A. 2004. Improvements to Linear Scan Register Allocation. Technical Report. University of California at Urbana-Champaign.

George, L. AND Appel, A. W. 1996. Iterated register coalescing. ACM Transactions on Programming Languages and Systems 18, 3, 300-324. 
Georges, A., Buytaert, D., And Eeckhout, L. 2007. Statistically rigorous Java performance evaluation. In Proceedings of the 22nd Annual ACM SIGPLAN Conference on Object Oriented Programming Systems and Applications (OOPSLA'07). ACM, New York, NY, 57-76.

Gupta, R., Soffa, M. L., AND Ombres, D. 1994. Efficient register allocation via coloring using clique separators. ACM Trans. Program. Lang. Syst. 16, 3, 370-386.

Hack, S. AND Goos, G. 2006. Optimal register allocation for SSA-form programs in polynomial time. Inf. Process. Lett. 98, 4, 150-155.

HACK, S. AND Goos, G. 2008. Copy coalescing by graph recoloring. In Proceedings of the ACM SIGPLAN 2008 Conference on Programming Language Design and Implementation (PLDI'08). ACM, New York, NY, 227-237.

JIKEs RVM. 2011. Homepage. Retrieved from http://jikesrvm.org/.

Jonathan Lee, K., PAlsberg, J., AND Pereira, F. M. 2007. Aliased register allocation for straight-line programs is NP-complete. In Automata, Languages and Programming. 680-691.

Kotzmann, T., Wimmer, C., Mössenböck, H. E., Rodriguez, T., Russell, K., and Cox, D. 2008. Design of the Java HotSpot ${ }^{\text {TM }}$ client compiler for Java 6. ACM Trans. Archit. Code Optim. 5, 1, 1-32.

LatTNeR, C. 2009. The LLVM Compiler Infrastructure. Retrieved from http://llvm.org/.

Lueh, G.-Y., Gross, T., And AdL-Tabatabai, A.-R. 2000. Fusion-based register allocation. ACM Trans. Program. Lang. Syst. 22, 431-470. Issue 3.

PARK, J. AND Moon, S.-M. 1998. Optimistic register coalescing. In Proceedings of the 1998 International Conference on Parallel Architectures and Compilation Techniques (PACT'98). J.-L. Gaudiot, Ed., 196-204.

Pereira F. M. AND PALsBerg, J. 2005. Register allocation via coloring of chordal graphs. In Proceedings of the 3rd Asian Symposium on Programming Languages and Systems (APLAS'05). 315-329.

Pereira F. M. ANd Palsberg, J. 2008. Register allocation by puzzle solving. In Proceedings of the ACM SIGPLAN 2008 Conference on Programming Language Design and Implementation (PLDI'08). ACM, New York, NY, 216-226.

Pereira F. M. AND PAlsberg, J. 2009. SSA Elimination after register allocation. In Proceedings of the 18th International Conference on Compiler Construction (CC'09). Springer-Verlag, Berlin, 158-173.

Pereira F. M. Q. And Palsberg, J. 2010. Punctual coalescing. In Proceedings of the 19th Joint European Conference on Theory and Practice of Software, International Conference on Compiler Construction (CC'10/ETAPS'10). Springer-Verlag, Berlin, 165-184.

Poletto, M. AND SARKAR, V. 1999. Linear scan register allocation. ACM Transactions on Programming Languages and Systems 21, 5, 895-913.

RAmsey, N. AND Holloway, G. 2004. A generalized algorithm for graph-coloring register allocation. In Proceedings of the ACM SIGPLAN 2004 Conference on Programming Language Design and Implementation (PLDI'04). ACM, New York, NY, 277-288.

Rong, H. 2009. Tree register allocation. In Proceedings of the 42nd Annual IEEE/ACM International Symposium on Microarchitecture (MICRO'09). ACM, New York, NY, 67-77.

SARKAR, V. AND BARIK, R. 2007. Extended linear scan: An alternate foundation for global register allocation. In Proceedings of the 16th International Conference on Compiler Construction (CC'07).

Thammanur, S. AND Pande, S. 2004. A fast, memory-efficient register allocation framework for embedded systems. ACM Trans. Program. Lang. Syst. 26, 6, 938-974.

The Standard Performance Evaluation Corporation. 2006. SPEC CPU2006 Benchmarks. Retrieved from http://www.spec.org/cpu2006/.

Traub, O., Holloway, G. H., And Smith, M. D. 1998. Quality and speed in linear-scan register allocation. In Proceedings of the ACM SIGPLAN 1998 Conference on Programming Language Design and Implementation (PLDI'98). 142-151.

Wimmer, C. AND Franz, M. 2010. Linear scan register allocation on SSA form. In Proceedings of the Annual IEEE/ACM International Symposium on Code Generation and Optimization (CGO'10). ACM, New York, NY, 170-179.

Wimmer, C. AND MössenBöck, H. 2005. Optimized interval splitting in a linear scan register allocator. In Proceedings of the 1st ACM/USENIX International Conference on Virtual Execution Environments (VEE'05). ACM, New York, NY, 132-141.

Zhang, K., Zhang, T., AND PANDE, S. 2004. Binary translation to improve energy efficiency through post-pass register re-allocation. In Proceedings of the 4th ACM International Conference On Embedded Software (EMSOFT'04). ACM, New York, NY, 74-85.

Received June 2013; revised September 2013; accepted November 2013 